\title{
Global turbulence features across marginality and nonlocal pedestal-core interactions
}

\author{
L. Villard ${ }^{1}$, B.F. McMillan ${ }^{2}$, E. Lanti ${ }^{1}$, N.Ohana ${ }^{1}$, A. Bottino ${ }^{3}$, \\ A. Biancalani ${ }^{3}$, I. Novikau ${ }^{3}$, S. Brunner ${ }^{1}$, O. Sauter ${ }^{1}$, \\ N. Tronko ${ }^{3}$, A. Mishchenko ${ }^{4}$ \\ ${ }^{1}$ Swiss Plasma Center, EPFL, CH-1015 Lausanne, Switzerland \\ ${ }^{2}$ Center for Fusion, Space and Astrophysics, University of Warwick, UK \\ ${ }^{3}$ Max-Planck Intitute for Plasmaphysik, Garching, Germany \\ ${ }^{4}$ Max-Planck Intitute for Plasmaphysik, Greifswald, Germany \\ E-mail: laurent.villard@epfl.ch
}

\begin{abstract}
Spatially non-local aspects of turbulent transport in tokamak plasmas are examined with global gyrokinetic simulations using the ORB5 code. Inspired by very accurate measurements in the TCV tokamak in L-mode, we initialize plasma profiles with constant logarithmic gradients in the core and constant linear gradients in the 'pedestal' $(\rho \in[0.8,1])$. The main finding is that transport in the core is strongly affected by the presence of pedestal gradients. This non-local pedestal-core coupling appears to be correlated with the appearance of repetitive avalanches that propagate across both pedestal and core regions. Below a certain threshold value in pedestal gradient, no well defined frequency is found for avalanches. Above this threshold, a well-defined frequency shows up, which roughly matches that of the local GAM frequency near the plasma edge and is thus well below the local GAM frequency in the core: this behaviour is very similar to the global coherent mode structure observed in TCV. Above this threshold in pedestal gradient, the core transport increases sharply: there is therefore a non-locality in marginality. The Probability Density Functions (PDFs) of density, temperature, temperature gradient and potential are found to have nearly Gaussian distributions, whereas the heat flux can have, in the presence of avalanches, a more or less strongly positiveley skewed PDF, which could be fitted by a log-normal distribution. The skewness of the heat flux is found to be radially and non-locally dependent: its value in the plasma core critically depends on the presence of gradients in the pedestal. The relation flux vs gradient is examined in detail. The local instantaneous flux vs gradient relation shows a hysteresis behaviour during an avalanche but no clear correlation, unlike the flux and zonal flow (ZF) shearing rate, which are anti-correlated: flux is higher when shearing rate is lower. This leads to corrugated time-averaged radial profiles of transport, heat and temperature gradient, with heat diffusivity having local maxima where ZF shearing rate goes to zero and temperature gradient has local minima. Finally, we show how the flux vs gradient relation can be analyzed locally for series of simulations with different averaged gradients.
\end{abstract}




\section{Introduction}

Non-local effects of turbulence in magnetized plasmas have been evidenced since some time already in global, first-principle-based gyrokinetic simulations of turbulence [1]. Non-local effects are responsible for the finite size (or finite $\rho *$ ) effects on the heat diffusivity in ITG turbulence [2, 3]. Here, $\rho *=\rho_{s} / a$, with $\rho_{s}$ the ion sound Larmor radius and $a$ the plasma minor radius. These studies were conducted using global gyrokinetic codes and all examined plasma profiles with finite gradients in the plasma core, typically for $\rho / a<0.8$, but flat profiles in the outer regions, $\rho / a>0.8$. In Ref.[3], ion heat transport was shown to scale as a function of an effective size parameter $\rho *^{e f f}=\rho_{s} / \Delta$, with $\Delta$ the radial extent of the plasma which is unstable for the ITG. Turbulence spreading [4] is a possible explanation of this result, with bursts of turbulence originating in unstable regions propagating into the linearly stable regions. In Ref.[5], non-locality was investigated focusing on the edge-core interplay.

In real experiments, the plasma gradients extend up to the edge. The logarithmic gradients, $R / L_{T}=R|\nabla T| / T$, with $R$ the plasma major radius, are typically higher in the edge than in the core. This is true even in L-mode discharges, as shown in particular in Ref.[6], where a series of dedicated experiments on TCV were carried out measuring the edge profiles with an unprecedented accuracy. More precisely, it appeared that the plasma profiles are characterized by a roughly constant $R / L_{T}$ between the sawtooth inversion radius and the edge of the 'pedestal' around $\rho / a=0.8$, and a roughly constant $\nabla T$ in the 'pedestal' region, typically $0.8<\rho / a<1$.

The present study is inspired from these TCV measurements and is a continuation of previous works [7]. Global gyrokinetic simulations with the ORB5 code [8, 9] are carried out in both core and pedestal regions, varying the gradients independently in these regions, with the aim of detecting spatially non-local effects on ITG turbulence. Such global simulations, with temperatures that strongly vary from the core to the edge (up to a factor of about 20 for the cases considered in this paper), are particularly challenging, because they require high resolution and long times. Fortunately, the ORB5 code has recently been completely refactored and made more efficient thanks to several algorithmic implementation improvements, in particular for its massive parallelization [10, 11].

There seems to be a relation between spatially non-local transport and the occurence of avalanches. These are intermittent bursts that propagate radially and are responsible for carrying part of the turbulent fluxes. It was shown in global gyrokinetic simulations [12, 13, 14] that avalanches propagate in radial zones, with a direction of

propagation that depends on the sign of the time-averaged shearing rate of the $\vec{E} \times \vec{B}$ zonal flow $(\mathrm{ZF})$. The plasma appears thus to self-organize in a flow structure with radially corrugated temperature gradients and heat transport.

In TCV experiments, a radially coherent propagating feature was observed on temperature and density fluctuations using various diagnostics [15, 16]. The propagation spans both core and edge. Originally thought to be a Geodesic Acoustic Mode (GAM) 
[17], it actually shows a frequency that matches that of the GAM at the edge, but is well below the GAM frequency in the core. Thus, the possibility is that this feature might be due to, or affected by, nonlinear effects. In global gyrokinetic simulations the presence of these regular radially propagating oscillations was confirmed in some cases [7, 18]. We

shall see that the presence of the coherent mode propagating down to the core depends on the presence of gradients in the pedestal, and is a nonlinear, avalanche-like feature, consistently with the conjecture proposed in Ref.[19].

Since a fraction of the turbulent heat flux is carried by these intermittent events, we shall obtain the PDFs of various fluctuating quantities (density, temperature, potential, heat flux) in order to characterize the turbulence. It will be seen that while the PDF of density, temperature and potential are very close to Gaussian, that of the heat flux can substantially deviate from it, with a positive skewness reflecting the presence of large events with a higher probability than for Gaussian. We shall also investigate how the skewness of the flux varies radially and whether non-local effects are showing up.

The remainder of the paper is organized as follows. In the next section the model and numerical simulation setup is introduced. In section 3 a series of simulations with exactly the same profiles in the core, but with different pedestal gradients is shown and analysed. In section 4 we examine the statistical properties of fluctuations. In section 5 we focus on the relation between heat flux and gradient in a regime of avalanches. A summary of main findings and outlook is given in section 6 .

\section{Physical model and numerical parameters}

Our main tool for the investigations is the multi-species, electromagnetic, global gyrokinetic code ORB5 [8, 9, 10] based on a Lagrangian Particle-In-Cell (PIC), finite elements approach. The equations used in the code have been obtained from a systematic variational formulation of dynamics, and the code has been thoroughly cross-verified against various codes, see e.g. Ref.[20]. In this work, we shall use the electrostatic approximation and assume Boltzmann electrons.

The ORB5 code includes a control variates $(\delta f)$ scheme and is truly global, both in the geometrical sense (the domain extends from the magnetic axis to the last closed flux surface) and in the physics sense, i.e. all plasma profiles are fully evolved consistently, similarly to a so-called 'full- $f$ ' code. (The polarization density, however, is linearized with respect to a background density $n_{0}$.)

Particular care has been put on the source terms. Since it is a PIC code, the statistical sampling error needs to be not only reduced, but also controlled unless the nonlinear simulations are quickly drown in noise. Various noise control schemes have been implemented in ORB5: a modified Krook operator, a coarse graining procedure, and a quadtree smoothing algorithm. Also, various heating schemes are included in the code, which can be run in 'gradient-driven' or 'flux-driven' mode.

For the kind of studies in this paper, in particular close to marginality, the results were found to be very sensitive to the way the source terms are implemented. In 
particular, if sources do not conserve desirable momenta of the total distribution function, it can result in both quantitative (heat transport modified by a factor of more than 2) and qualitative differences (e.g. disapearance of the coherent mode). Therefore, we shall use here a Krook operator modified such as to exactly conserve, in radial bins, the density, parallel momentum, and zonal flow residual phase space structures [21],

$$
S=-\gamma_{K} \delta f+S_{\text {corr }}
$$

with $S_{\text {corr }}$ such that

$$
\left\langle\int \mathrm{d} \vec{v} M_{i}\left(\gamma_{K} \delta f+S_{\text {corr }}\right)\right\rangle=0, i=1 . .3, \mathbf{M}=\left\{1, v_{\|},\left(\frac{v_{\|}}{B}-\left\langle\frac{v_{\|}}{B}\right\rangle_{b}\right)\right\},
$$

where $\langle$.$\rangle means a flux-surface average and <.>_{b}$ means a bounce-average. The 'correction term' $S_{\text {corr }}$ is obtained in the following way. Defining the matrix $S_{i j}$ and the vector $\delta S_{j}$ as:

$$
S_{i j}(s, t)=\left\langle\int \mathrm{d} \vec{v} M_{i} M_{j} f_{0}\right\rangle, \delta S_{j}(s, t)=\gamma_{K}\left\langle\int \mathrm{d} \vec{v} \delta f M_{j}\right\rangle,
$$

We solve the linear system $S_{i j} g_{j}=\delta S_{i}$ for the coeffs $g_{i}$ and we have:

$$
S_{\text {corr }}=\sum_{i=1}^{3} g_{i} f_{0} M_{i}
$$

The Krook operator does not conserve kinetic energy, and therefore the temperature will tend to relax to the initial profiles. It serves thus both purposes of a noise control and heating operator. The value of the coefficient $\gamma_{K}$ has to be chosen high enough so as to be effectively controlling the noise down to an acceptable level but small enough so as to not modify crucially the physics under investigation. This is empirically chosen and requires careful verification. Typically, its value is a few percent of the maximum linear growth rate, and we check that the results are not too sensitive to it.

Temperature and density profiles are chosen such as to reflect the TCV findings, namely different functional forms in the core and the pedestal. With the definition of the radial coordinate $\rho_{V}=\sqrt{V(\psi) / V\left(\psi_{a}\right)}$, where $V(\psi)$ is the volume enclosed by the magnetic surface $\psi=$ const, $\psi$ is the poloidal flux, and $\psi_{a}$ its value at the last closed flux surface, we specify:

$$
\begin{array}{ll}
T\left(\rho_{V}\right)=\min \left(T_{0}, T_{\text {ped }} \exp \left(-\kappa_{T}\left(\rho_{V}-\rho_{V, \text { ped }}\right)\right)\right) & \rho \leq \rho_{V, \text { ped }} \\
T_{1}\left(1-\mu_{T}\left(\rho_{V}-\rho_{V, \text { edge }}\right)\right) & \rho_{V, \text { ped }}<\rho \leq \rho_{V, \text { edge }}
\end{array}
$$

where $T_{0}, T_{1}, \rho_{V, \text { ped }}, \rho_{V \text {,edge }}, \kappa_{T}$ and $\mu_{T}$ are given input parameters and $T_{\text {ped }}=$ $T_{1}\left(1+\mu_{T}\left(\rho_{V \text {,edge }}-\rho_{V \text {,ped }}\right)\right)$. Density profiles are defined in a similar way, with parameters $n_{0}, n_{1}, \kappa_{n}$ and $\mu_{n}$.

Toroidal, magnetic, straight-field line coordinates are used for the finite element representation of perturbed fields: $\left(s, \theta_{*}, \varphi\right)$, where $s=\sqrt{\psi / \psi_{a}}, \theta_{*}$ is the poloidal coordinate and $\varphi$ the geometrical toroidal angle, such that $\vec{B} \cdot \nabla \varphi / \vec{B} \cdot \nabla \theta_{*}$ is constant on a magnetic surface. 

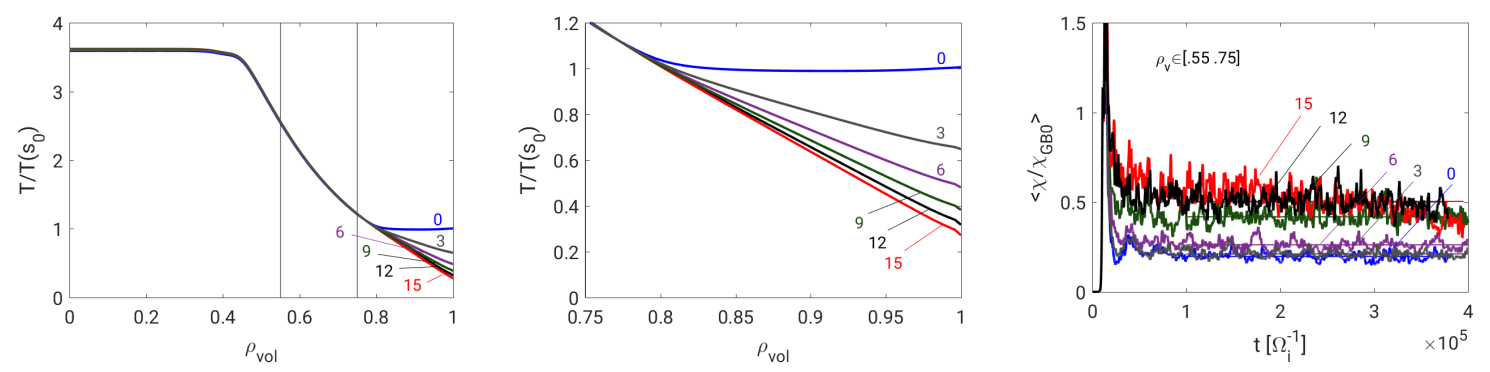

Figure 1. Left: $T$ profiles with various pedestal gradients. Middle: a zoom of the pedestal profiles. Right: effective ion heat diffusivity in the core vs time, for different pedestal gradients. the labels indicate the value of the $\mu_{T}$ parameter. The vertical lines indicate the region over which $\chi$ is averaged: $\rho_{v} \in[.55, .75]$.

Boundary conditions are the unicity condition for $\phi$ at the magnetic axis $(s=0)$ and $\phi=0$ at the plasma boundary $(s=1)$, which sets (unrealistically) turbulent field fluctuations to zero there. On the other hand, we do not impose $\delta f=0$ at the boundary. Instead, for numerical particles (markers) that leave the plasma, we put them back into the plasma at a position which would conserve the particle energy, magnetic moment and toroidal canonical momentum but put their weight to zero in order to avoid unphysical accumulation of perturbed density at the boundary.

The magnetic configuration is an axisymmetric ideal MHD equilibrium obtained with the CHEASE code [22] from a reconstructed equilibrium from the TCV experiment [6], with aspect ratio 3.64, an elongation 1.44, triangularity 0.2 . The $q$ profile is monotonic with $q_{0}=0.78, q_{a}=3.29$.

Unless specified otherwise, simulations are run with $N_{p}=256 \times 10^{6}, N_{s} \times N_{\theta *} \times N_{\varphi}=$ $256 \times 512 \times 256$. A field-aligned Fourier filter is applied with $n_{\max }=128, \Delta m=5$, a Krook coefficient $\gamma_{K}=1.4 \times 10^{-4}\left[\Omega_{i}\right]$. Simulation time is $t_{\mathrm{sim}}=4 \times 10^{5}\left[\Omega_{i}^{-1}\right] \approx$ $3000\left[a / c_{s, \text { ped }}\right]$, with $c_{s, \text { ped }}$ the ion sound speed at the pedestal position $\rho_{V \text {,ped }}$.

\section{Pedestal-core nonlocal coupling}

We consider a series of $T$ profiles defined by Eq.(5) with $\kappa_{T}=3.7, \rho_{V \text {,ped }}=0.8$, $\rho_{V \text {,edge }}=1$, i.e. the core profiles are kept identical, and vary the pedestal profiles by choosing various values for $\mu_{T}$ from 0 to 15 . The value of $\rho *$ at the top of the pedestal is $1 / 133$. In all simulations we keep the same density profile with $\kappa_{n}=2.3, \mu_{n}=5$ and $T_{e}=T_{i}$ everywhere.

Figure 1 shows the $T$ profiles used and the effective ion heat diffusivity in the core vs time for the various pedestal temperature profiles considered. Figure 2 shows the temperature gradient profiles, the $R / L_{T}$ profiles, and the time-averaged ion heat diffusivity vs radius. The striking feature is the strong dependence of core transport on the gradients in the pedestal. Looking at the data more carefully, it appears that there is some kind of threshold for the strength of this non-local effect: for a minor change in 

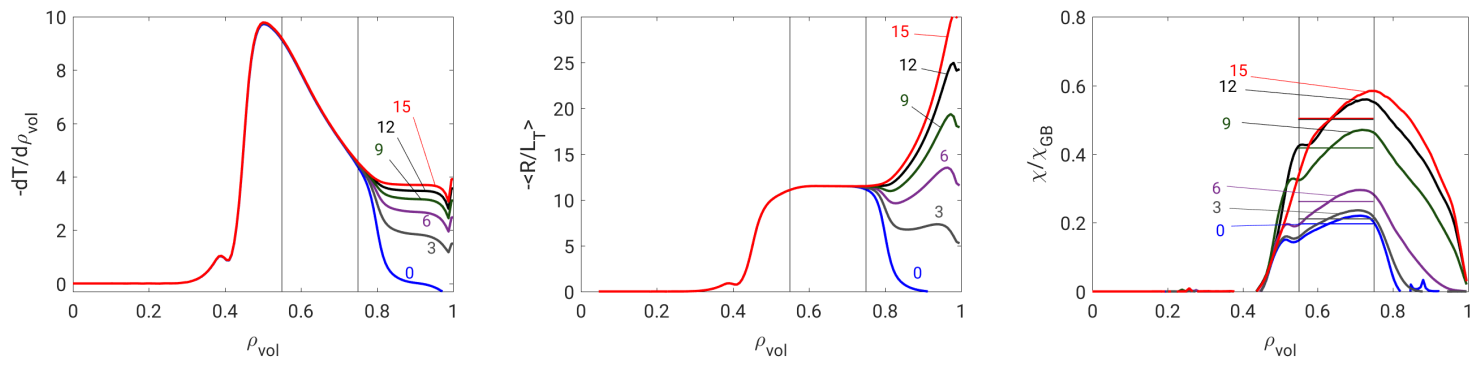

Figure 2. Left: $d T / d \rho_{v}$ profiles with various pedestal gradients. Middle: $R / L_{T}$ profiles. Right: effective ion heat diffusivity vs radius, time-averaged over the quasisteady-phase of the simulations, for different pedestal gradients. Labels and vertical lines: see Fig 1 .

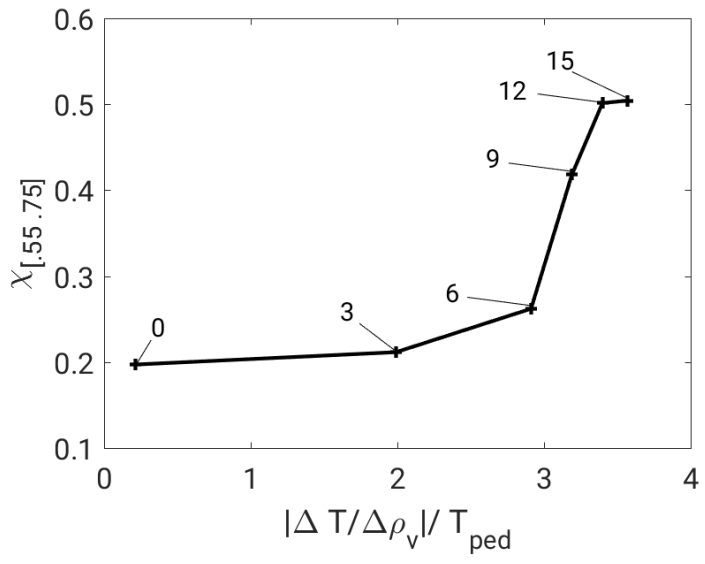

Figure 3. Time- and radius-averaged ion heat diffusivity $\chi$ in the core vs temperature gradient in the pedestal. Data point labels are values of $\mu_{T}$.
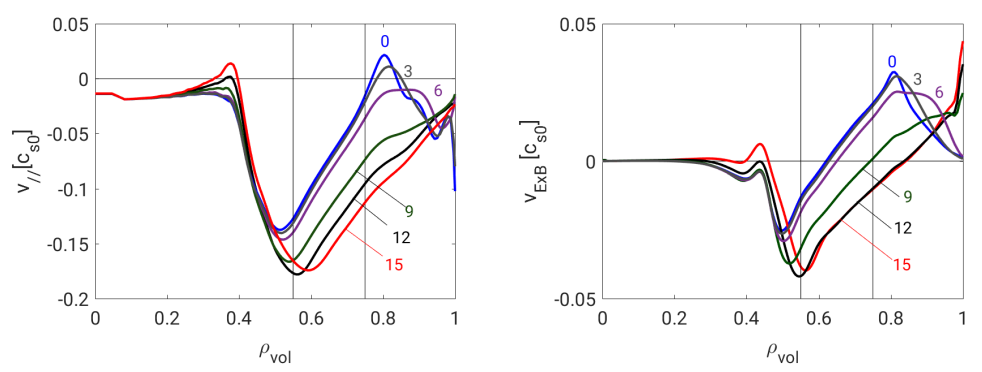

Figure 4. Left: radial profile of parallel flows. Right: radial profile of zonal $\vec{E} \times \vec{B}$ flows. Time-averaged data over the quasi-steady phase of the simulations. Labels and vertical lines: see Fig[1. 

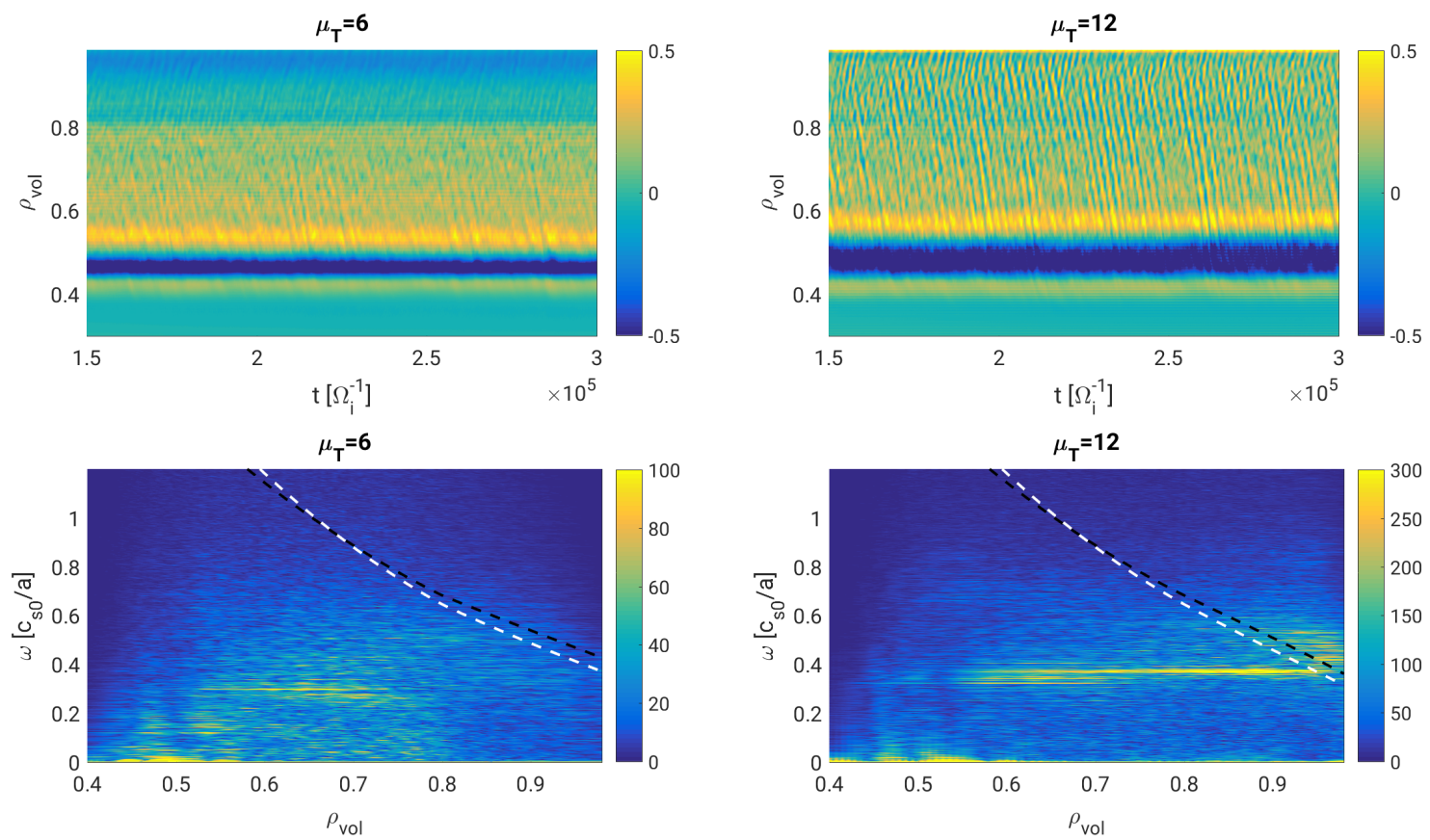

Figure 5. Top row: contours of $\vec{E} \times \vec{B}$ shearing rate vs radius and time. Bottom row: frequency spectra. Left column: below the transition, $\mu_{T}=6$. Right column: above the transition, $\mu_{T}=12$. The white dashed lines are the local GAM frequencies as defined in Eq.(20) of Ref. 23]. The black dashed lines are the local GAM frequencies as in Ref. 24.

the pedestal profiles ( $\mu_{T}$ from 6 to 9 ), there is a strong effect on $\chi$. For higher values of the pedestal gradient, $\mu_{T}>12$, the effect seems to saturate.

This is confirmed when we plot in Fig. 3 the radially-averaged $\left(\rho_{v} \in[.55, .75]\right)$, time-averaged $\chi$ in the core as a function of the normalized pedestal gradient $\left(1 / T_{\text {ped }}\left|d T / d \rho_{v}\right|_{\text {ped }}\right.$. The sharp transition is clearly visible: there seems thus to be a critical pedestal gradient value above which transport in the core is abruptly enhanced.

In order to understand if the turbulent regime has changed below and above the critical pedestal gradient, we examine the behaviour of flows. Figure 4 shows the timeaveraged parallel and zonal $\vec{E} \times \vec{B}$ flows vs radius for various pedestal temperature profiles. The shearing rates in the core do not seem to be much affected, but there is a reversal of flow direction in the pedestal, which occurs at the same critical pedestal gradients, between $\mu_{T}=6$ and $\mu_{T}=9$, as the core transport sharp transition, see Fig 3 .

The fluctuating part of the ZFs exhibits a remarkable transition change in behaviour. We show in Fig.5, top row, contours of the zonal $\vec{E} \times \vec{B}$ shearing rate vs radius and time, below (left, $\mu_{T}=6$ ) and above (right, $\mu_{T}=12$ ) transition. Above the critical pedestal gradient, avalanches are triggered at a regular pace and propagate radially at constant velocity from the edge up to the plasma core. Frequency spectra of the zonal $\vec{E} \times \vec{B}$ shearing rate confirm this, Fig.5, bottom row: below the critical pedestal gradient, there is no well-defined frequency for avalanches. Above critical 
pedestal gradient, a radially coherent oscillation appears. Its frequency coincides with the local GAM frequency at the plasma edge. Being constant over radius, the frequency is well below the local GAM frequency in the core. The frequency of these regular avalanches matches well that of the observations on TCV of a 'coherent mode', even though the physical model used here is too simple to justify a quantitative comparison. For more realistic simulations, including the trapped electron response, more relevant to the TCV cases, see Ref. [25], where a similar coherent mode is observed at roughly the same frequency, i.e. nearly matching the edge GAM frequency. For a detailed study of the linear GAM dynamics including kinetic electrons and relevant for the Asdex-Upgrade tokamak, see Ref.[19].

In order to determine the linear or nonlinear nature of the 'coherent mode', we have conducted a numerical experiment in which the turbulent field perturbations $\delta \phi_{n \neq 0}$ were abruptly turned off in the middle of quasi-steady phase: the coherent mode immediately disappears from $n=0 \vec{E} \times \vec{B}$ flows signals. Thus, the process of excitation of the coherent mode is through nonlinear coupling of turbulence to the zonal component of $\vec{E} \times \vec{B}$ flows. It is therefore justified to identify the presence of the coherent mode as a manifestation of regular avalanches.

Thus, avalanches appear as the vehicle through which transport is 'delocalized' from the pedestal region to the core. However, care must be taken before a too simplistic explanation is given: in the TEM cases studied in Ref.[25], avalanches propagate mostly outward, so it is not obvious to find a causality relation between the edge GAM frequency and the avalanche coherent frequency. The following sections focus on the role of avalanches on statistical properties of fluxes and gradients, and on the relation between these.

\section{Statistical properties}

We examine here how the fluctuations statistics behave as a function of radius, for a case $\kappa_{T}=3.1, \mu_{T}=12$. Figure 6 shows the probability density functions for various quantities: temperature, density and potential all show normal (Gaussian) PDFs, whereas the heat flux exhibits a long tail, representative of large events. This reflects in a positive skewness. The PDF of heat flux can be fitted by a log-normal distribution, which is indicated by the dashed line. A log-normal distribution can result from the product of independent random variables, unlike the Gaussian PDF which results from the sum of independent random variables. Such behaviour (normal PDFs for gradients, but skewed PDFs for fluxes) were also observed in flux-driven simulations of ITG turbulence in Ref.[26].

Taken at different radii, the skewness of the temperature, density and potential all show a Gaussian statistics and thus zero skewness. The heat flux skewness, however, shows a strong radial dependence, increasing as we move into the core. This could be related to the direction of avalanche propagation, which is inward in this case, although this would need verification in cases where avalanches would propagate outward. 

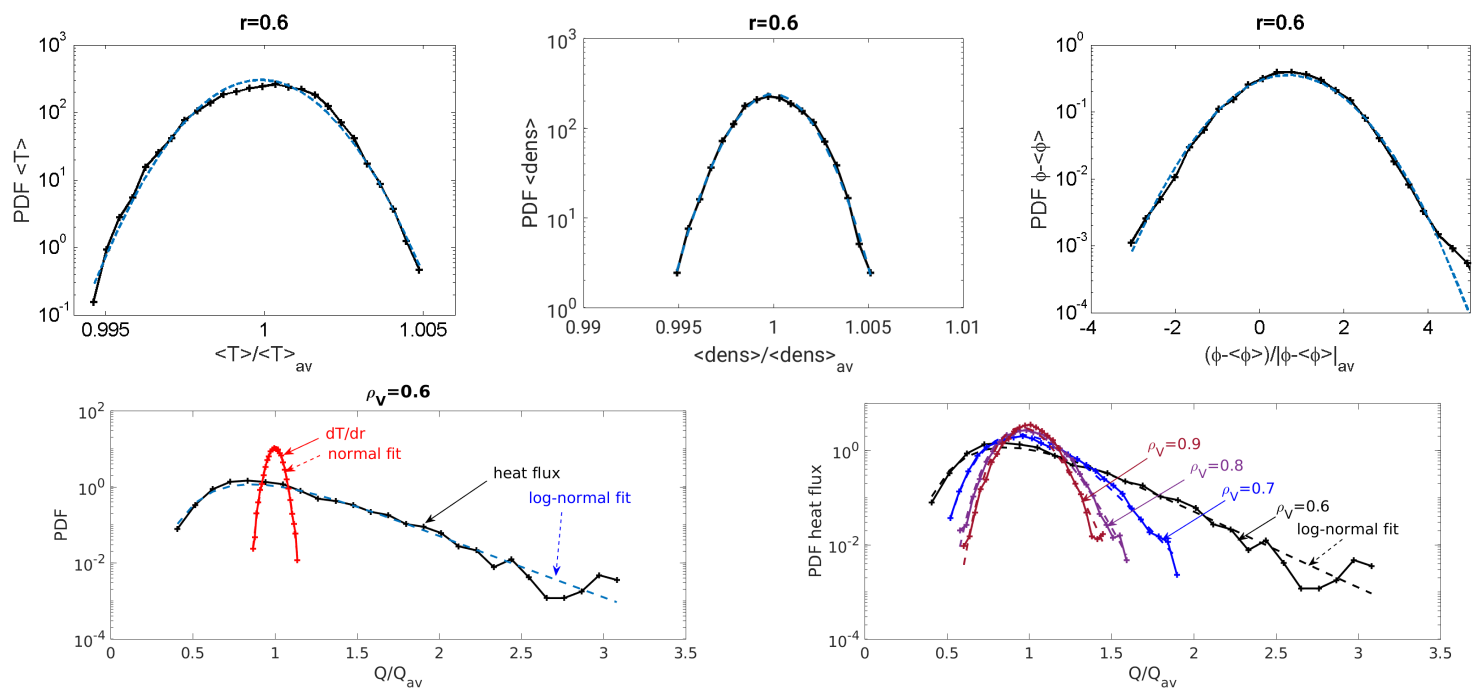

Figure 6. Top, from left to right: PDFs of temperature, density and non-zonal perturbed potential, at $\rho_{v}=0.6$. Bottom, left: PDFs of heat flux and of temperature gradient at $\rho_{V}=0.6$. Right: PDFs of heat flux at various radial positions. Simulation with core and pedestal gradients, $\kappa_{T}=3.1, \mu_{T}=12$.

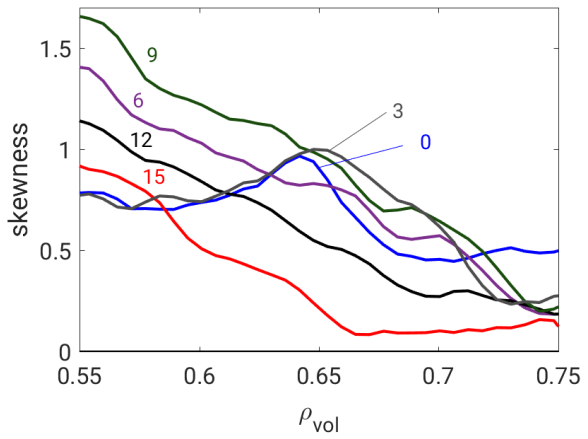

Figure 7. Skewness of the PDF of the turbulent power heat flux as a function of radius in the core region, for various pedestal gradients, and the same value of core gradient, $\kappa_{T}=3.7$. Labels indicate the values of $\mu_{T}$.

The comparison of the statistical properties for different values of the pedestal gradient shows that mostly the temperature, density and potential fluctuations are invariably well described by Gaussian PDFs. The skewness of the heat flux as a function of radius, on the other hand, shows an interesting behaviour below or above the 'critical' $\mu_{T}$, as shown in Fig.7. Below the critical $\mu_{T}$ (0 or 3), the skewness is almost constant between $\rho_{v}=0.55$ and $\rho_{v}=0.65$. For larger values of $\mu_{T}$ the skewness is monotonically decreasing function of radius, but, interestingly, it is does not have a monotonic behaviour with respect to the value of pedestal gradient: the skewness first increases, reaching a maximum for $\mu_{T}=9$, which is at the upper edge of the sharp transport transition observed in Fig 3 , and then decreases. 

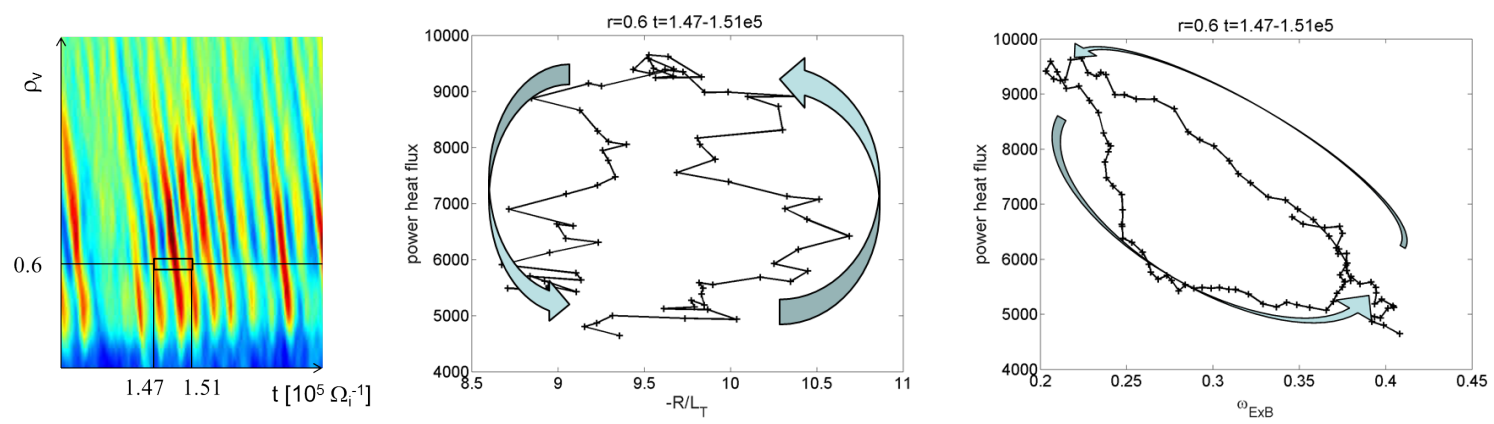

Figure 8. Left: contours of heat flux vs radius and time, zooming around a single avalanche event (small box). Middle: instantaneous local heat flux vs $R \mathrm{E}_{T}$. The arrows indicate the direction of time evolution. Right: instantaneous local heat flux vs $\mathrm{ZF} \vec{E} \times \vec{B}$ shearing rate.
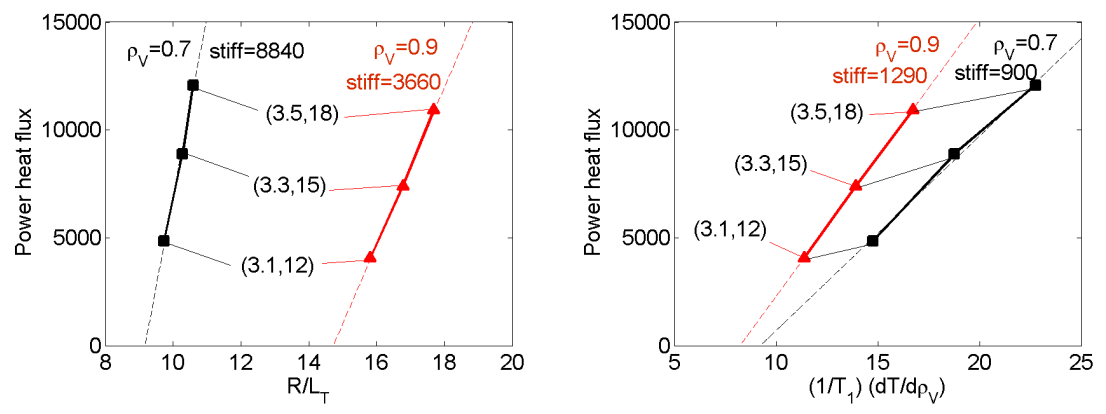

Figure 9. Left: time-averaged heat power vs $R / L_{T}$. Right, time-averaged heat power vs $\left(1 / T_{1}\right)\left(d T / d \rho_{v}\right)$, in the core $\left(\rho_{v}=0.7\right)$ and in the pedestal $\left(\rho_{v}=0.9\right)$. The data points labels indicate the corresponding values of $\left(\kappa_{T}, \mu_{T}\right)$. The unit power for this study is $n_{1} m_{i} \rho_{s, 1}^{2} c_{s, 1}^{3}$, where the subscript 1 indicates values at the edge $\left(\rho_{V}=1\right)$.

\section{Avalanches and flux vs gradient relation}

Focusing now on what happens during the passage of an avalanche at a given radius, Fig,8, we can observe a non-trivial relation between the local, instantaneous heat flux and temperature gradient: it is characterized by a hysteresis, the flux first increasing at high gradient value, then the gradient decreasing at high flux value, then the flux decreasing at low gradient value. This behaviour repeats at each avalanche. So, the heat flux appear to lead the evolution of the gradient.

Remarkably, the local, instantaneous heat flux shows a decreasing function of the $\mathrm{ZF} \vec{E} \times \vec{B}$ shearing rate, with some hysteresis as well. This is at least consistent with the paradigm of turbulence suppression by sheared zonal flows.

The time-averaged flux vs gradient relationship at different radial positions shows a non-trivial behaviour as well. This was best seen in a larger plasma size (ITER simulations in Ref.[14]): global effects lead to corrugated profiles of temperature gradient and ZF shearing rate ('staircase', [12]). Transport is maximal at radial positions where 
the ZF $\vec{E} \times \vec{B}$ shearing rate is minimal and results in a local minimum of the temperature gradient. This leads to a decreasing heat transport vs gradient.

Taking the radial average of the time-averaged fluxes and gradients, and considering different global simulations with various averaged gradients in both core and pedestal, leads to a more familiar, monotonically increasing $\chi$ vs gradient relation. The global, time-averaged, radially averaged flux vs gradient relation 'looks like' a local one. But, as we have seen, this does not mean that transport is local. Coming back to the TCVlike simulations, considering various core and edge gradients [7], taking the time- and radius-averaged (over, repsectively, the core and pedestal regions), we can analyze the flux vs gradient relation 'as if it were local' and conclude to a stiffness and a critical normalized logarithmic gradient $R / L_{T}$ in the core much larger than in the pedestal, see Fig 9, left. We note that multiplying the power by a factor of 2.5 leads to only a modest increase of the core logarithmic gradient $\kappa_{T}$ from 3.1 to 3.5, i.e. by about $13 \%$ only, whereas the linear gradient in the pedestal $\mu_{T}$ is increased from 12 to 18, i.e.by $50 \%$. This is very much in line with experimental findings on TCV, see Figs 8 and 10 of Ref. [6]. Interestingly, when represented as function of the normalized linear gradient, $\left(1 / T_{1}\right)\left(d T / d \rho_{V}\right)$, the stiffness and the critical gradient in the core and in the pedestal are almost identical, Fig 9 , right.

\section{Conclusions}

The radially non-local nature of ITG turbulent transport has been evidenced in a series of global gyrokinetic simulations. In particular, a strong relation exists between transport in the core and the presence of gradients in the pedestal. The behaviour of core transport as a function of pedestal gradient shows a critical behaviour (Fig 3).

The statistical properties of fluctuations show normal (Gaussian) PDFs for temperature, temperature gradient, density and potential, whereas the heat flux can be strongly positively skewed. This skewness also exhibits a non-local behaviour (Fig 7).

The non-locality is associated with the presence of avalanches. Above the (nonlocal) criticality, these show a repetitive, regular pattern, propagating at constant speed from the pedestal to the core, with a frequency matching that of the local GAM frequency at the edge but much below the local GAM frequency in the core (Fig 5 . very similarly to TCV observations [15, 16].

The leading role of $\vec{E} \times \vec{B} \mathrm{ZFs}$ for the avalanche propagation has been analysed in detail. The local, instantaneous flux vs gradient relation appears to be regulated by the local, instantaneous $\vec{E} \times \vec{B}$ zonal shearing rate.

Taking appropriate time averages, the transport in the core is much stiffer than in the pedestal, when measured as a function of the logarithmic gradient $R / L_{T}$, but not so when measured as a function of the linear gradient $d T / d \rho_{v}$. The effective critical $R / L_{T}$ is much higher in the pedestal than in the core, but the effective critical $d T / d \rho_{v}$ is almost the same. (Fig, 9). These findings are consistent with experimental observations on TCV [6]. 
These simulations were all done with the adiabatic electron assumption, which is known to lead to largely underestimated values of power fluxes and no particle flux. Future works will address the same non-locality investigations but with the inclusion of kinetic electron dynamics, in order to approach more realistic values of fluxes, so as to allow for a more quantitative comparison with experimental observations.

\section{Acknowledgements}

Discussions with G. Dif-Pradalier and X. Garbet are gratefully acknowledged. This work has been carried out within the framework of the EUROfusion Consortium and has received funding from the Euratom research and training programme 2014-2018 under grant agreement No 633053, project EnR AWP17-ENR-MFE-CEA-02 (TNT). The views and opinions expressed herein do not necessarily reflect those of the European Commission. This work was partly supported by the Swiss National Science Foundation. This work was supported by a grant from the Swiss National Supercomputing Centre (CSCS) under project ID s760. Additional computations were performed on the Marconi platform at CINECA in Italy.

\section{References}

[1] Garbet X, Idomura Y, Villard L and Watanabe T H 2010 Nucl. Fusion 50043002

[2] Lin Z, Ethier S, Hahm T S and Tang W M 2002 Phys. Rev. Lett. 88195004

[3] McMillan B F, Lapillonne X, Brunner S, Villard L, Jolliet S, Bottino A, Görler T and Jenko F 2010 Phys. Rev. Lett 105155001

[4] Hahm T S, Diamond P H, Lin Z, Itoh K and Itoh S I 2004 Plasma Phys. Control. Fusion 46 A323

[5] Dif-Pradalier G, Caschera E, Ghendrih P, Asahi Y, Donnel P, Garbet X, Grandgirard V, Latu G, Norscini C and Sarazin Y 2017 Plasma Fusion Res. 121203012

[6] Sauter O et al 2014 Phys. Plasmas 21055906

[7] Villard L, McMillan B F, Sauter O, Hariri F, Dominski J, Merlo G, Brunner S and Tran T M 2014 J. Phys. Conf. Series $\mathbf{5 6 1} 012022$

[8] Jolliet S, Bottino A, Angelino P, Hatzky R, Tran T M, McMillan B F, Sauter O, Idomura Y and Villard L 2007 Comput. Phys.Commun. 177409

[9] Bottino A, Vernay T, Scott B, Brunner S, Hatzky R, Jolliet S, McMillan B F, Tran T M and Villard L 2011 Plasma Phys. Control. Fusion 53124027

[10] Lanti E et al 2018 Comput. Phys. Commun., submitted

[11] Ohana N, Jocksch A, Lanti E, Tran T M, Brunner B, Gheller C, Hariri F and Villard L $2016 \mathrm{~J}$. Phys. Conf. Series $\mathbf{7 7 5} 012010$

[12] Dif-Pradalier G, et al 2010 Phys. Rev. E 82025401

[13] McMillan B F, Hill P, Bottino A, Jolliet S, Vernay T and Villard L 2011 Phys. Plasmas 18112503

[14] Villard L, Angelino P, Bottino A, Brunner S, Jolliet S, McMillan B F, Tran T M and Vernay T 2013 Plasma Phys. Control. Fusion 55074017

[15] de Meijere C A et al 2014 Plasma Phys. Control. Fusion 56072001

[16] Huang Z, Coda S, Merlo G, Brunner S, Villard L, Labit B, Theiler C and the TCV team 2018 Plasma Phys. Control. Fusion 60034007

[17] Winsor N, Johnson J L and Dawson J M 1968 Phys. Fluids 112448

[18] Merlo G et al 2018 Plasma Phys. Control. Fusion 60034003

[19] Novikau I, et al 2017 Phys. Plasmas 24122117 
[20] Tronko N, Bottino A, Görler T, Sonnendrücker E, Told D and Villard L 2017 Phys. Plasmas 24 056115

[21] McMillan B F, Jolliet S, Tran T M, Villard L, Bottino A and Angelino P 2008 Phys. Plasmas 15 052308

[22] Lütjens H, Bondeson A and Sauter O. 1996Comput. Phys. Commun. 97219

[23] Gao Z. 2010 Phys. Plasmas 17092503

[24] Angelino P et al 2008 Phys. Plasmas 15062306

[25] Lanti E, McMillan B F, Brunner S, Ohana N and Villard L 2018 J. Phys. Conf. Series, submitted

[26] Idomura I and Nakata M 2014 Phys. Plasmas 21020706 